\title{
Chiral Arene Ligand as Stereocontroller for Asymmetric C-H Activation
}

\author{
Hao Liang, Weicong Guo, Junxuan Li, Jijun Jiang and Jun Wang*
}

\section{Affiliations:}

Key Laboratory of Bioinorganic and Synthetic Chemistry of Ministry of Education, School of Chemistry, Guangdong Key Laboratory of Chiral Molecule and Drug Discovery, Sun Yat-Sen University, Guangzhou, 510006, P. R. China.

*Corresponding author. Email: wangjun23@mail.sysu.edu

\begin{abstract}
:
Development of chiral ligands is the most fundamental task in metal-catalyzed asymmetric synthesis. In the last 60 years, various kinds of ligands have been sophisticatedly developed. However, it remains a long-standing challenge to develop practically useful chiral $\eta^{6}$-arene ligands, thereby seriously hampering the asymmetric synthesis promoted by arene-metal catalysts. Herein, we report the design and synthesis of a class of facilely tunable, $C_{2}$ symmetric chiral arene ligands derived from [2.2]paracyclophane. Its ruthenium(II) complexes have been successfully applied in the enantioselective $\mathrm{C}-\mathrm{H}$ activation to afford a series of axially chiral biaryl compounds (up to 99\% yield and 96\% ee). This study not only lays chemists' longstanding doubts about whether it is possible to use chiral arene ligand to stereocontrol asymmetric C$\mathrm{H}$ activation, but also opens up a new avenue to achieve asymmetric $\mathrm{C}-\mathrm{H}$ activation.
\end{abstract}

\section{Keywords:}

ruthenium, chiral arene ligand, [2.2]paracyclophane, asymmetric $\mathrm{C}-\mathrm{H}$ activation, axially chiral 


\section{Main Text:}

Transition-metal-catalyzed asymmetric $\mathrm{C}-\mathrm{H}$ activation is of great scientific significance and practical values in modern organic chemistry. ${ }^{1}$ It aims to convert simple and easily available feedstocks to highvalue-added enantiomerically enriched chiral compounds via inert $\mathrm{C}-\mathrm{H}$ bond activation and subsequent functionalization, which features high atom- and step-economy. Over the past decades, palladium, ${ }^{2,3}$ rhodium $^{4,5}$ and ruthenium ${ }^{6,7}$ turn out to be three most prominent transition metals to catalyze C-H activation. Nevertheless, in terms of their related asymmetric catalyzed $\mathrm{C}-\mathrm{H}$ activation, ruthenium has lagged far behind palladium ${ }^{1,8,9}$ and rhodium ${ }^{1,10}$, and actually largely undeveloped except for the classical C-H bonds insertion reactions. ${ }^{11}$ As early as 2000, Murai and coworkers reported an atropselective alkylation of naphthyl pyridines and naphthyl quinolines by ruthenium-catalyzed C-H/olefin coupling (up to $49 \%$ ee). ${ }^{12}$ It is not until recently that some breakthroughs have been made by taking advantage of chiral transient directing group ${ }^{13,14}$ or employing chiral carboxylic acid ${ }^{15-17}$, in which $\left[\mathrm{RuCl}_{2}(p \text {-cymene) }]_{2}\right.$ was used as catalyst. However, despite these encouraging achievements, the above strategies may find restricted applications because of their specific mechanistic features.

Given the fact that currently the ruthenium catalysts applied in C-H activation are mostly derived from $\left[\mathrm{RuCl}_{2}(p \text {-cymene })\right]_{2}$, it would be natural to imagine that development of effective chiral arene ligands should be a universally viable strategy to realize a vast number of ruthenium(II)-catalyzed asymmetric C$\mathrm{H}$ activation (Figure 1a). However, it is a formidable challenge, and no precedent has been accomplished so far. The possible reasons are as follows. Firstly, it is well known that in many catalytic processes arene ligands dissociate from metals, ${ }^{18}$ which may largely discourage researchers from developing chiral arene ligands for asymmetric catalysis since such dissociation would result in catalyst deactivation or nonstereoselective background reactions. Secondly, preparation of ruthenium complexes with a chiral arene ligand is not a trivial task. ${ }^{19-26}$ Most of their syntheses are associated with handling air-sensitive ruthenation reagents, using large excess of chiral arene ligands, or conducting chiral resolution of ruthenium complexes. Consequently, their availability, diversity, and tunability are greatly restricted. Therefore, it may be difficult to identify a catalyst possessing a satisfied chiral environment for asymmetric catalysis. In addition, another difficulty for designing effective chiral arene ligands is that most of the privileged chiral scaffolds (e.g., BINOL, SPINOL) contain arene motifs, which may interfere the ligand synthesis, and arouse site- and stereo-selectivitive issues during metal complexation process. Herein, we wish to report a class of easily tunable chiral arene ligand, and the first example of rutheniumcatalyzed asymmetric $\mathrm{C}-\mathrm{H}$ activation enabled by a chiral arene ligand.

In order to conquer the afore-mentioned challenges, we rationally designed a class of $C_{2}$ symmetric planar chiral pseudo-ortho-disubstituted [2.2]paracyclophanes as chiral arene ligands, in which the benzene rings constituted the chiral skeletons are considered to be used as $\eta^{6}$-coordinating groups. As shown in Figure 1b, structure I, although there are two benzene rings and thereby four benzene faces in each ligand, owing to the unique structure of [2.2]paracyclophane, only the two external benzene faces of [2.2]paracyclophanes are accessible by coordinating metals. Moreover, these two accessible benzene faces are related by a $C_{2}$ symmetric axis and therefore homotopic, which can completely avoid the generation of regio- and stereo-isomers during complexation. To create a good chiral environment around the metal center, we speculated that the substituents on [2.2]paracyclophane would be suitably as alkyl groups. As depicted in Figure 1b, structure II, the functional group R' at lower place was anticipated to turn towards the metal side owing to the prominent steric hindrance posed by the upper non-coordinated benzene ring. And the chiral environment around the metal should be convenient to tune by varying the R' substituent. In addition, the arene metal complexes were expected to have improved stability because of the protection of the coordinated benzene ring from any possible nucleophilic attack by the upper non-coordinated 
benzene ring, ${ }^{27}$ as well as by the unique transannular electronic interactions between the two benzene rings in the paracyclophane structures (Figure 1b, structures III and IV). ${ }^{28,29}$

As shown in Scheme 1a, the designed ligands were synthesized from the enantiomerically pure aldehyde 1, which was prepared from commercially available racemic 4,12-dibromo[2.2]paracyclophane either by a literature protocol ${ }^{30}$ or our newly developed method (see Supplementary Materials). Reacting 1 with Grignard reagent produced the corresponding alcohols $\mathbf{2 a - c}$, which were reduced with triethylsilane in the presence of $\mathrm{BF}_{3} \cdot \mathrm{Et}_{2} \mathrm{O}$ to give ligands 3a-c. For ligands $\mathbf{3 b}$-c , the products were contaminated with a small amount of dehydrated products. Pure products could be obtained by simply treating the crude products with $\mathrm{H}_{2}$ in the presence of $\mathrm{Pd} / \mathrm{C}$, under which conditions the alkenes could be fully reduced into the desired products. Besides, ligands 3d-f with a different substituting pattern were synthesized. Firstly, the aldehyde 1 was oxidized to the corresponding ester $\mathbf{4}$ by iodine in methanol. Then it was transformed to the tertiary alcohols $\mathbf{2 d - f}$ by treating with Grignard reagent or organolithium reagent. Finally, the ligands 3d-f were generated in $45-82 \%$ yield by following the same afore-mentioned reductive deoxygenation protocol.

Subsequently, as shown in Scheme 1b, starting from the arene ligands 3a-f, we synthesized an array of chiral arene ruthenium dichlorides (6a-f, 50-90\% yields) by modifying a literature procedure described by Boekelheide and coworkers. ${ }^{31,32}$ Then, their catalytic performance was evaluated. Encouragingly, all the complexes proved catalytically active for the $\mathrm{C}-\mathrm{H}$ activation reaction of $\mathrm{N}$ methoxyl benzamide $\mathbf{7 a}$ with the alkyne $\mathbf{8 a},{ }^{33}$ affording the axially chiral biaryl product 9aa in almost quantitative yields (Figure 2). It was found that the enantioselectivity of the reaction was significantly influenced by the alkyl substituents on the chiral arene ligands, which was fully consistent with our design (Figure 1b, structure II). In general, the catalysts with branched alkyl substituents on benzene rings gave superior enantioselectivity. The catalyst $\left(S_{p}\right)$-6d bearing two isopropyl groups provided the best enantioselectivity of $91 \%$ ee. However, extending the branched methyl groups of isopropyl to ethyl $\left(\left(R_{p}\right)\right.$ 6e) or n-butyl groups $\left(\left(S_{p}\right)\right.$-6f $)$ could not further enhance the enantioselectivity. With the optimal catalyst $\left(S_{p}\right)$-6d, we further investigated various substituted amides $\mathbf{7}$ and alkynes $\mathbf{8}$ as reactants. To our delight, excellent yields and enantioselectivities were observed for most of the substrates possessing substituents with diverse electronic properties as well as in different substitution fashions (Figure 2). The absolute configuration of product 9aa and 9ac were assigned to be $S$ by comparison of specific optical rotations (for details, see Supplementary Materials).

To learn more details about how alkyl substituents affected chiral environment around metal center in catalyst, we resorted to single crystal X-ray diffraction technology. Despite all attempts to grow single crystals of ruthenium dichloride failed, we successfully obtained single crystals of two bis-arene ruthenium complexes $\left(R_{p}\right)$-5a and $\left(R_{p}\right)$-5g. X-Ray crystallography revealed that the distances between the two centroids of benzene rings of paracyclophane motif were $2.993 \AA$ and $2.923 \AA$ respectively for $\left(R_{p}\right)$ 5a and $\left(R_{p}\right)$-5g (Figure 3a). They were manifestly shorter than that of [2.2]paracyclophane (3.101 $⿱$ A), ${ }^{34}$ implying the existence of transannular electronic interaction that should benefit the catalyst stability. To understand why better enantioselectivity was achieved by the isopropyl substituted catalyst than that by the ethyl substituted one ( $91 \%$ ee $v s 65 \%$ ee), steric map analysis ${ }^{35}$ was performed. However, they exhibited very similar stereochemical environment around ruthenium (Figure 3). By spectating the crystal structures, we found the distance of the two benzylic carbon was $4.397 \AA$ for the ethyl substituted ligand and $4.918 \AA$ for the isopropyl substituted ligand, and the dihedral angle between the two benzene rings was $0.662^{\circ}$ for the former and $7.754^{\circ}$ for the latter. It suggests a strong steric interaction exist between the two isopropyl groups, which restrains the rotation of isopropyl group at the metal-coordinated benzene ring and hence results in a more confined chiral environment around the metal center. 
In conclusion, a class of facilely tunable chiral arene ligand derived from [2.2]paracyclophane has been designed and synthesized. Owing to its unique structure, only two of the four benzene faces are accessible by metal for complexation. Moreover, these two accessible benzene faces are related by a $C_{2}$ symmetry axis and thus homotopic, hence avoiding generation of stereoisomers during complexation. Significantly, with this chiral arene ligand, a ruthenium-catalyzed enantioselective $\mathrm{C}-\mathrm{H}$ activation has been successfully accomplished, furnishing a series of axially chiral biaryl compounds in up to $99 \%$ yield and up to $96 \%$ ee. It worth noting that the described ligand represents the first example of chiral [2.2]paracyclophane ligand that coordinates to metals by its arene ring rather than heteroatoms to generate chiral $\eta^{6}$-arene metal complexes. Moreover, this work also represents the first case of asymmetric C-H activation stereocontrolled by a chiral $\eta^{6}$-arene ligand. It not only lays chemists' longstanding doubts about whether it is possible to use chiral arene ligand to stereocontrol asymmetric $\mathrm{C}-\mathrm{H}$ activation, but also opens up a new and appealing avenue to achieve catalytic asymmetric $\mathrm{C}-\mathrm{H}$ activation that is now largely dominated by palladium and rhodium catalysis. Apart from its charming scientific significance, ruthenium-catalyzed asymmetric $\mathrm{C}-\mathrm{H}$ activation is also of great practical values in view of the much lower price of ruthenium than those of palladium and rhodium. Further application of chiral [2.2]paracyclophane complexes in other asymmetric $\mathrm{C}-\mathrm{H}$ activation reactions are underway.

\section{References}

1 Newton, C. G., Wang, S. G., Oliveira, C. C. \& Cramer, N. Catalytic Enantioselective Transformations Involving C-H Bond Cleavage by Transition-Metal Complexes. Chem. Rev. 117, 8908-8976 (2017).

2 Lyons, T. W. \& Sanford, M. S. Palladium-Catalyzed Ligand-Directed C-H Functionalization Reactions. Chem. Rev. 110, 1147-1169 (2010).

3 He, J., Wasa, M., Chan, K. S. L., Shao, Q. \& Yu, J.-Q. Palladium-Catalyzed Transformations of Alkyl C-H Bonds. Chem. Rev. 117, 8754-8786 (2017).

4 Rej, S. \& Chatani, N. Rhodium-Catalyzed C(sp2)- or C(sp3)-H Bond Functionalization Assisted by Removable Directing Groups. Angew. Chem. Int. Ed. 58, 8304-8329 (2019).

5 Colby, D. A., Bergman, R. G. \& Ellman, J. A. Rhodium-catalyzed C-C bond formation via heteroatom-directed C-H bond activation. Chem. Rev. 110, 624-655 (2010).

6 Arockiam, P. B., Bruneau, C. \& Dixneuf, P. H. Ruthenium(II)-Catalyzed C-H Bond Activation and Functionalization. Chem. Rev. 112, 5879-5918 (2012).

7 Singh, K. S. Recent Advances in C-H Bond Functionalization with Ruthenium-Based Catalysts. Catalysts 9, 173 (2019).

8 Shao, Q., Wu, K., Zhuang, Z., Qian, S. \& Yu, J.-Q. From Pd(OAc)2 to Chiral Catalysts: The Discovery and Development of Bifunctional Mono-N-Protected Amino Acid Ligands for Diverse C-H Functionalization Reactions. Acc. Chem. Res. 53, 833-851 (2020).

9 Yang, K., Song, M., Liu, H. \& Ge, H. Palladium-catalyzed direct asymmetric C-H bond functionalization enabled by the directing group strategy. Chem. Sci. 11, 12616-12632 (2020).

10 Mas-Roselló, J., Herraiz, A. G., Audic, B., Laverny, A. \& Cramer, N. Chiral Cyclopentadienyl Ligands: Design, Syntheses, and Applications in Asymmetric Catalysis. Angew. Chem. Int. Ed. 60, 13198-13224 (2021). 
11 Wu, W.-T., Yang, Z.-P. \& You, S.-L. in Asymmetric Functionalization of C-H Bonds, Ch. Asymmetric C-H Bond Insertion Reactions, 1-66 (The Royal Society of Chemistry, 2015).

12 Kakiuchi, F., Le Gendre, P., Yamada, A., Ohtaki, H. \& Murai, S. Atropselective alkylation of biaryl compounds by means of transition metal-catalyzed $\mathrm{C}-\mathrm{H} /$ olefin coupling. Tetrahedron: Asymmetry 11, 2647-2651 (2000).

13 Li, Z.-Y. et al. Ruthenium-Catalyzed Enantioselective C-H Functionalization: A Practical Access to Optically Active Indoline Derivatives. J. Am. Chem. Soc. 141, 15730-15736 (2019).

14 Li, G., Liu, Q., Vasamsetty, L., Guo, W. \& Wang, J. Ruthenium(II)-Catalyzed Asymmetric Inert $\mathrm{C}-\mathrm{H}$ Bond Activation Assisted by a Chiral Transient Directing Group. Angew. Chem. Int. Ed. 59, 3475-3479 (2020).

15 Dhawa, U., Connon, R., Oliveira, J. C. A., Steinbock, R. \& Ackermann, L. Enantioselective Ruthenium-Catalyzed C-H Alkylations by a Chiral Carboxylic Acid with Attractive Dispersive Interactions. Org. Lett. 23, 2760-2765 (2021).

16 Huang, L.-T. et al. Ruthenium(II)/Chiral Carboxylic Acid Catalyzed Enantioselective C-H Functionalization of Sulfoximines. Synthesis, DOI: 10.1055/a-1588-0072.

17 Zhou, T. et al. Efficient Synthesis of Sulfur-Stereogenic Sulfoximines via Ru(II)-Catalyzed Enantioselective C-H Functionalization Enabled by Chiral Carboxylic Acid. J. Am. Chem. Soc. 143, 6810-6816 (2021).

18 Rigby, J. H. \& Kondratenko, M. A. in Transition Metal Arene $\pi$-Complexes in Organic Synthesis and Catalysis (ed E. Peter Kündig) Ch. Arene Complexes as Catalysts, 181-204 (Springer Berlin Heidelberg, 2004).

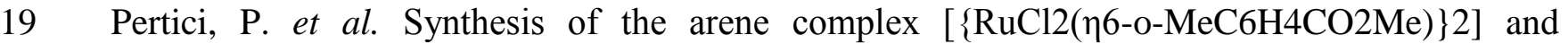
separation of its diastereomeric (-)(S)-1-phenylethylamine adducts. J. Chem. Soc., Dalton Trans., 315-320 (1988).

20 Pertici, P. et al. Preparation and resolution of chiral areneruthenium(II) complexes. J. Organomet. Chem. 466, 221-231 (1994).

21 Heinemann, F., Klodwig, E., Knoch, F., Wündisch, M. \& Zenneck, U. Chiral arene ruthenium

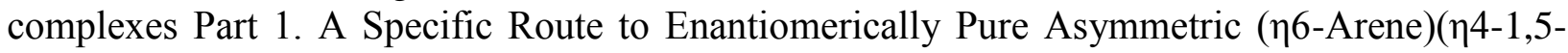
cyclooctadiene)Ru(0) Complexes. Chem. Ber. 130, 123-130 (1997).

22 Bodes, G., Heinemann, F. W., Marconi, G., Neumann, S. \& Zenneck, U. Chiral arene ruthenium

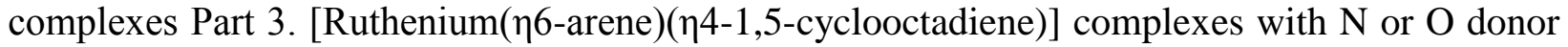
functions in the aren side chain. J. Organomet. Chem. 641, 90-101 (2002).

23 Vieille-Petit, L., Therrien, B. \& Süss-Fink, G. Chiral Arene Ruthenium Complexes: Synthesis and Molecular Structure of the Enantiopure Cluster Cation (S)[H3Ru3 $\{\mathrm{C} 6 \mathrm{H} 5[\mathrm{CH}(\mathrm{CH} 3) \mathrm{CH} 2 \mathrm{OH}]\}(\mathrm{C} 6 \mathrm{Me} 6) 2(\mathrm{O})]$. Eur. J. Inorg. Chem. 2003, 3707-3711 (2003).

24 Pinto, P. et al. Diastereoselective Synthesis of Arene Ruthenium(II) Complexes Containing Chiral Phosphetane-Based Tethers. Organometallics 25, 2607-2616 (2006).

25 Reiner, T. et al. Phenylalanine - a biogenic ligand with flexible $\eta 6$ - and $\eta 6: \kappa 1$-coordination at ruthenium(ii) centres. Dalton Trans. 42, 8692-8703 (2013). 
26 Pototskiy, R. A., Boym, M. A., Nelyubina, Y. V. \& Perekalin, D. S. Synthesis of Ruthenium Catalysts with a Chiral Arene Ligand Derived from Natural Camphor. Synthesis, DOI: 10.1055/a1668-2075.

27 Davies, S. G., Green, M. L. H. \& Mingos, D. M. P. Nucleophilic addition to organotransition metal cations containing unsaturated hydrocarbon ligands: A survey and interpretation. Tetrahedron 34, 3047-3077 (1978).

28 Cram, D. J. \& Wilkinson, D. I. Macro Rings. XXIII. Carbonylchromium Complexes of Paracyclophanes and Model Compounds1. J. Am. Chem. Soc. 82, 5721-5723 (1960).

29 Maekawa, M. et al. Syntheses and structural characterization of [2.2]paracyclophane complexes of rhodium and iridium supported by diene ligands. Inorg. Chim. Acta 344, 143-157 (2003).

30 Morisaki, Y., Hifumi, R., Lin, L., Inoshita, K. \& Chujo, Y. Practical Optical Resolution of Planar Chiral Pseudo-ortho-disubstituted [2.2]Paracyclophane. Chem. Lett. 41, 990-992 (2012).

31 Laganis, E. D. et al. A study of the synthesis and properties of ruthenium complexes of [2n]cyclophanes. Organometallics 1, 1415-1420 (1982).

32 Swann, R. T., Hanson, A. W. \& Boekelheide, V. Ruthenium complexes of [2n]cyclophanes. A general synthesis of bis( $76-[2 n]$ cyclophane)ruthenium(II) compounds and related chemistry. J. Am. Chem. Soc. 108, 3324-3334 (1986).

33 Wang, F. et al. Rhodium(III)-Catalyzed Atroposelective Synthesis of Biaryls by C-H Activation and Intermolecular Coupling with Sterically Hindered Alkynes. Angew. Chem. Int. Ed. 59, 1328813294 (2020).

34 Wolf, H., Jorgensen, M. R. V., Chen, Y.-S., Herbst-Irmer, R. \& Stalke, D. Charge density investigations on [2,2]-paracyclophane - in data we trust. Acta Cryst. B71, 10-19 (2015).

35 Falivene, L. et al. Towards the online computer-aided design of catalytic pockets. Nat. Chem. 11, 872-879 (2019).

Acknowledgments: National Natural Science Foundation of China (Grant 21971263).

Author contributions: J.W. conceived the project. H.L., W.G., J.L. and J.J. designed and conducted the experiments. J.W. directed the research, and all authors wrote the manuscript.

Competing interests: The Authors declare no competing finical interests.

Data and materials availability: Crystallographic data are available free of charge from the Cambridge Crystallographic Data. All other data are available in the main text or the supplementary materials. 
(a) Evolvement of chiral arene-ruthenium catalyst for asymmetric $\mathrm{C}-\mathrm{H}$ activation

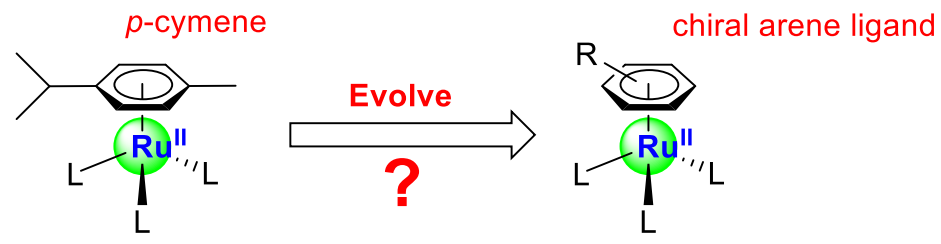

(b) Rational design of chiral arene ligand based on [2.2]-paracyclophane

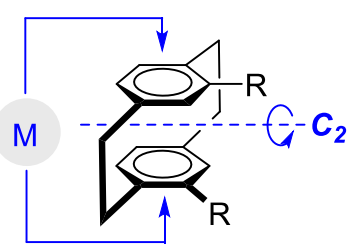

(I)

Homotopic arene faces ensure formation of single metal complex

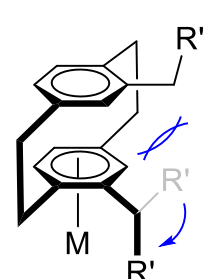

(II)

Steric group turns to metal side to ensure good chiral environment

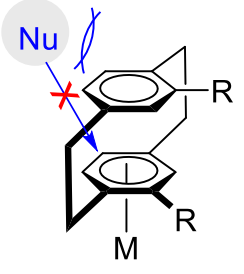

(III)

The coordinated arene is protected from nucleophilic attack

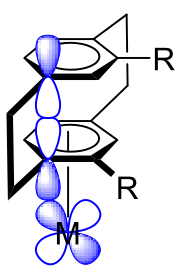

(IV)

Transannular interaction enhances stability of complex

Figure 1. Research background and design of chiral arene ligand. 
(a) Synthesis of chiral arene ligands
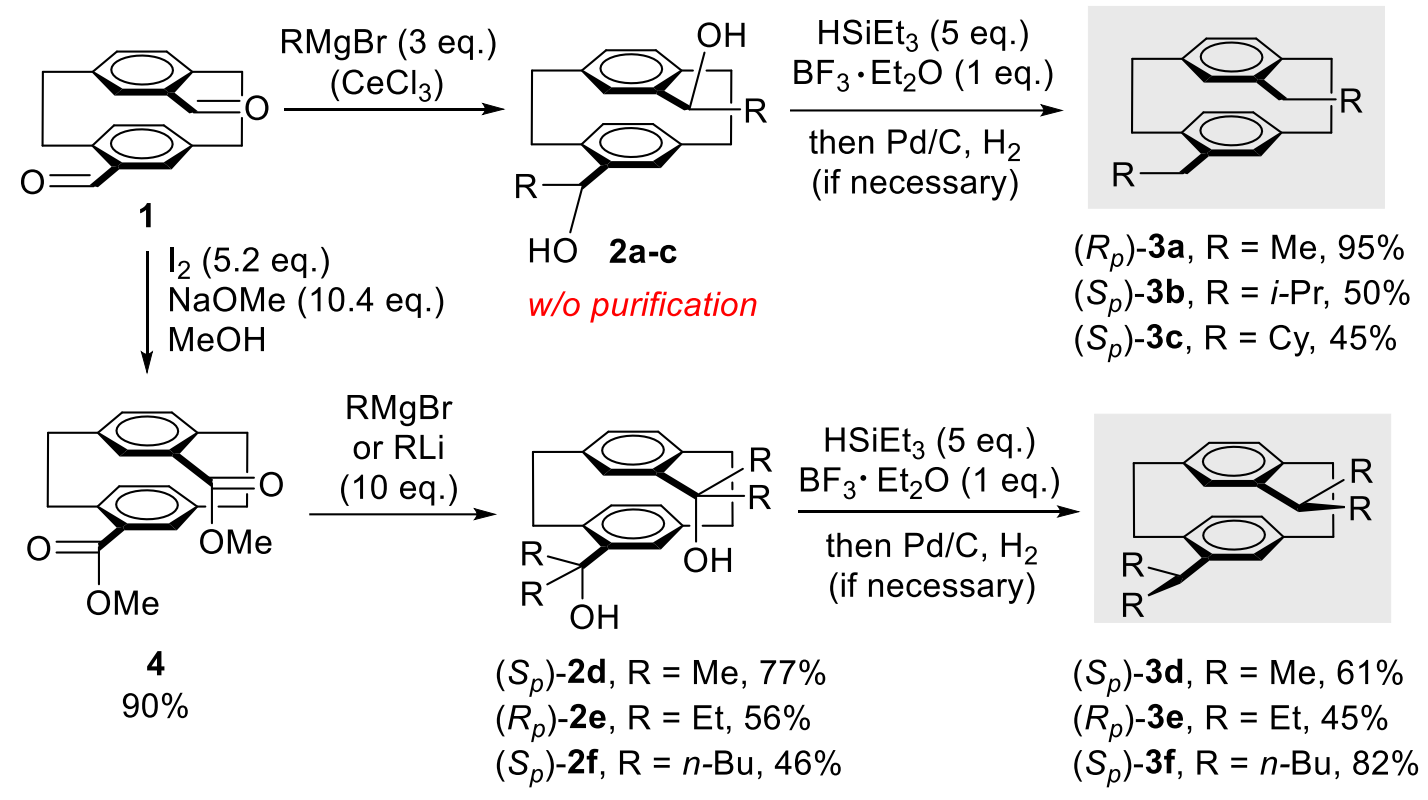

(b) Synthesis of chiral arene-ruthenium complexes

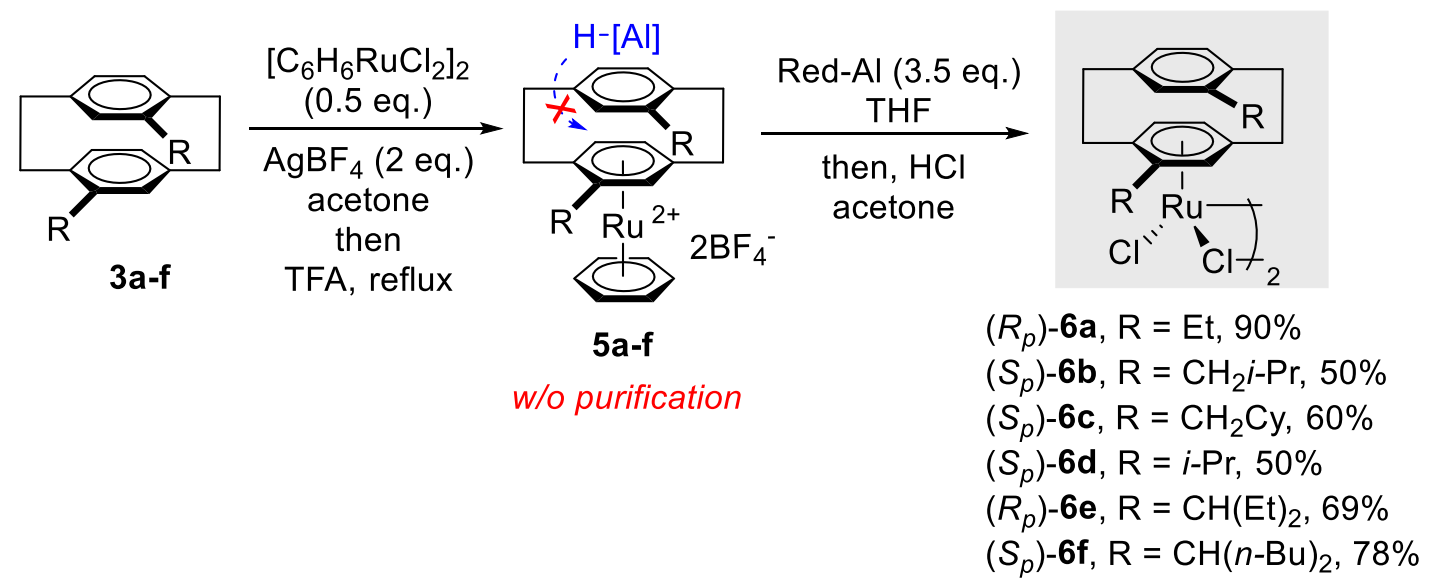

Scheme 1. Synthesis of chiral arene ligands and their ruthenium complexes. 


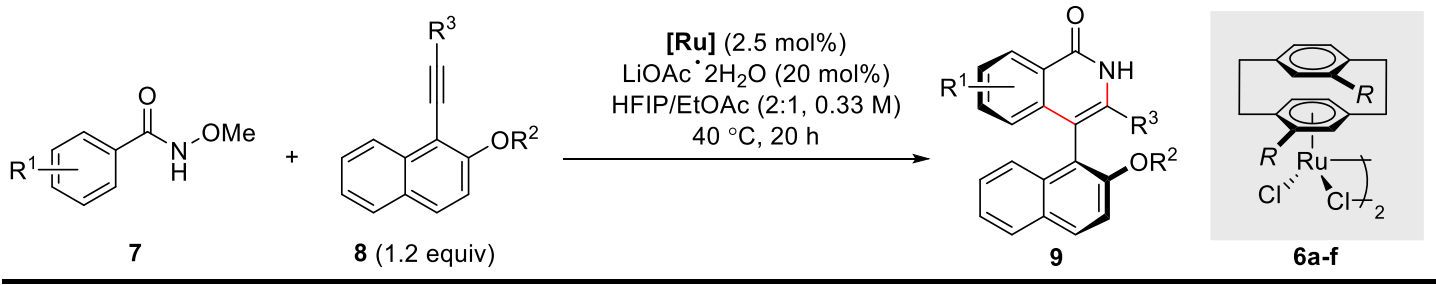

Catalyst screening ${ }^{a}$
$\left(S_{p}\right)-6 \mathbf{b},>95 \%$ yield, $84 \%$ ee
$\left(S_{p}\right)-6 \mathbf{c},>95 \%$ yield, $82 \%$ ee
$\left(S_{p}\right)-6 \mathbf{d},>95 \%$ yield, $91 \%$ ee
$\left(R_{p}\right)-6 \mathbf{e},>95 \%$ yield, $90 \%$ ee
$\left(S_{p}\right)-6 \mathbf{6 f},>95 \%$ yield, $90 \%$ ee

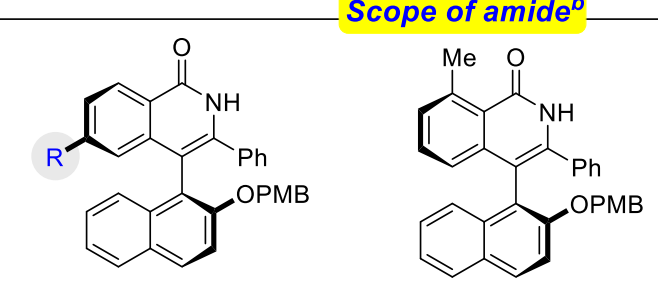

9aa, $\mathrm{R}=\mathrm{H}, 99 \%$ yield, $91 \%$ ee 9ba, $\mathrm{R}=\mathrm{Me}, 92 \%$ yield, $91 \%$ ee 9ca, $\mathrm{R}=\mathrm{Ph}, 94 \%$ yield, $93 \%$ ee 9da, $\mathrm{R}={ }^{t} \mathrm{Bu}, 96 \%$ yield, $76 \%$ ee 9ea, $R=O M e, 99 \%$ yield, $95 \%$ ee 9fa, $R=F, 94 \%$ yield, $94 \%$ ee 9ga, $\mathrm{R}=\mathrm{Cl}, 92 \%$ yield, $95 \%$ ee 9ha, $\mathrm{R}=\mathrm{Br}, 47 \%$ yield, $94 \%$ ee 9ia, $\mathrm{R}=\mathrm{CO}_{2} \mathrm{Me}, 97 \%$ yield, $96 \%$ ee 9ja, $\mathrm{R}=\mathrm{CF}_{3}, 72 \%$ yield, $95 \%$ ee 9ka, $\mathrm{R}=\mathrm{NO}_{2}, 71 \%$ yield, $95 \%$ ee 9la, $\mathrm{R}=\mathrm{CN}, 39 \%$ yield, $96 \%$ ee $90 \mathrm{a}, 87 \%$ yield, $91 \%$ ee<smiles>CCOc1ccc2ccccc2c1-c1c(-c2ccccc2)[nH]c(=O)c2c(C)cc(C)cc12</smiles>

9na, $98 \%$ yield, $90 \%$ ee<smiles>CCOc1ccc2ccccc2c1-c1c(-c2ccccc2)[nH]c(=O)c2cc(C)cc(C)c12</smiles>

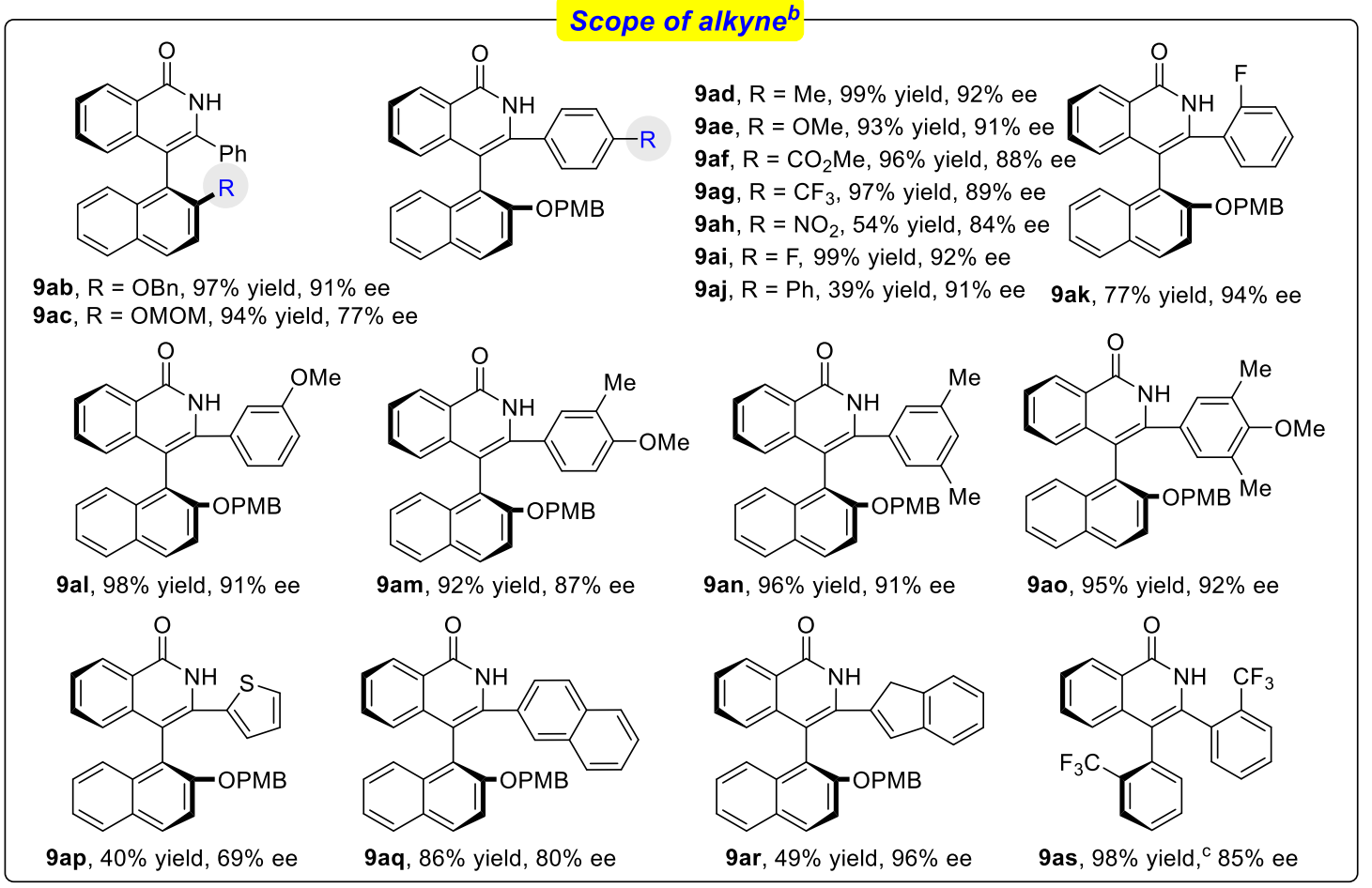

Figure 2. Application of chiral arene ruthenium catalysts in asymmetric $\mathbf{C}-\mathrm{H}$ activation. ${ }^{a} \mathbf{7 a}(0.05$ $\mathrm{mmol}), \mathbf{8 a}(0.06 \mathrm{mmol}), \mathbf{6}(2.5 \mathrm{~mol} \%), \mathrm{LiOAc} \cdot 2 \mathrm{H}_{2} \mathrm{O}(20 \mathrm{~mol} \%)$ in HFIP/EtOAc (2/1, v/v, $\left.0.15 \mathrm{~mL}\right)$ at 40 ${ }^{\circ} \mathrm{C}$ for $20 \mathrm{~h}$. Yields were determined by ${ }^{1} \mathrm{H}$ NMR analysis using $\mathrm{CH}_{2} \mathrm{Br}_{2}$ as internal standard. ${ }^{b} 7(0.1 \mathrm{mmol})$, $8(0.12 \mathrm{mmol}),\left(S_{p}\right)$-6d $(2.5 \mathrm{~mol} \%), \mathrm{LiOAc} \cdot 2 \mathrm{H}_{2} \mathrm{O}(20 \mathrm{~mol} \%)$ in HFIP/EtOAc $(2 / 1, \mathrm{v} / \mathrm{v}, 0.3 \mathrm{~mL})$ at $40{ }^{\circ} \mathrm{C}$ for $20 \mathrm{~h}$. Regioselectivity >20:1 for all cases. Isolated yields were reported. ${ }^{c} \mathrm{HFIP}$ as solvent. 
(a) Single crystal structures and steric maps

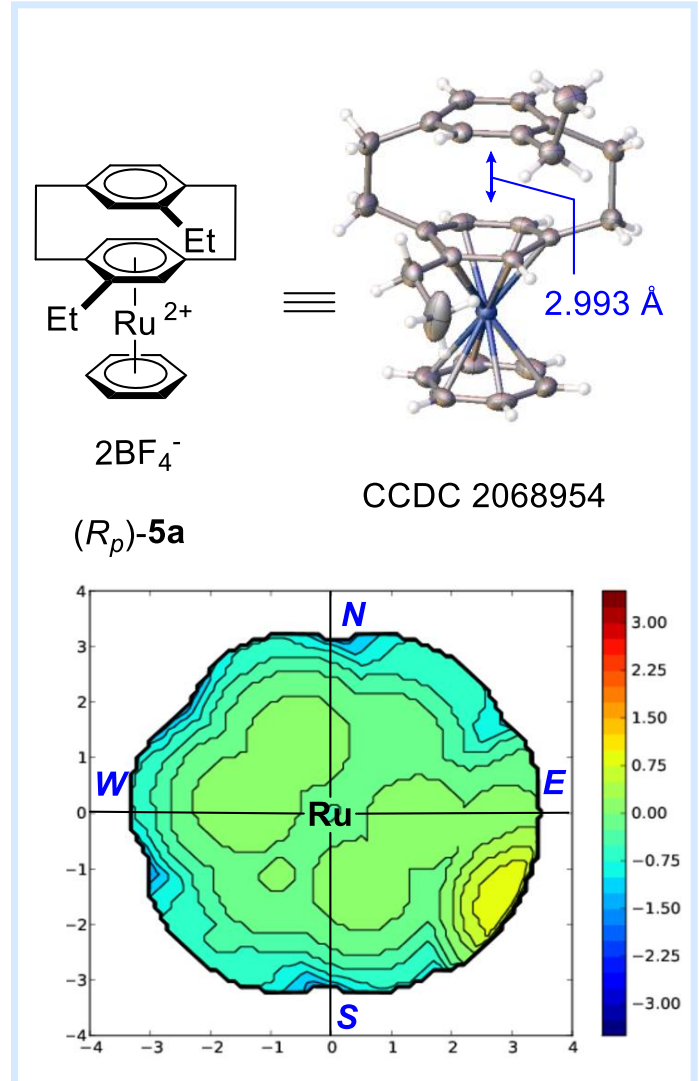

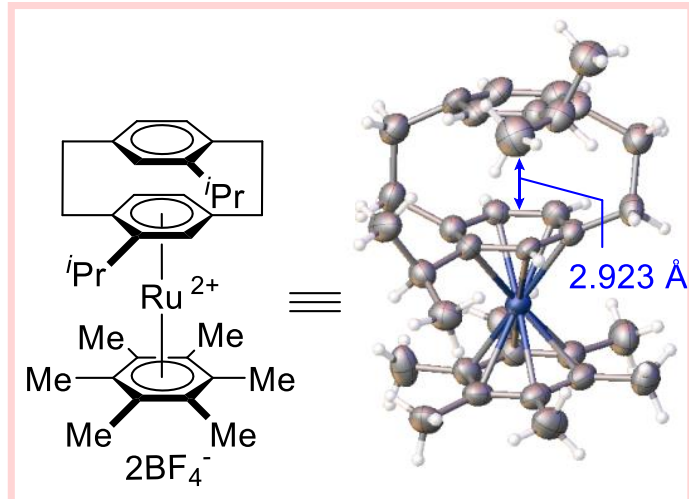

$\left(R_{p}\right)-5 g$

CCDC 2101186

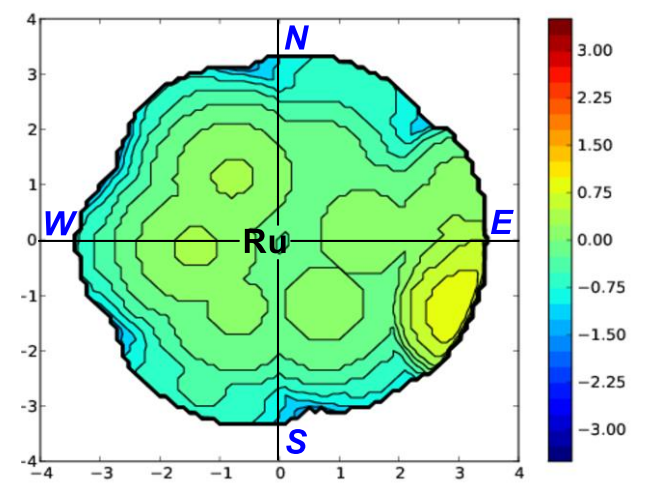

(b) Characteristic distances and angles

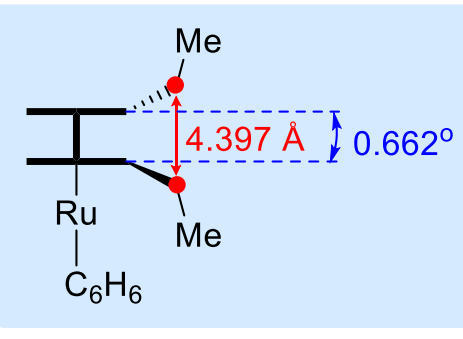

$\left(R_{p}\right)-5 a$
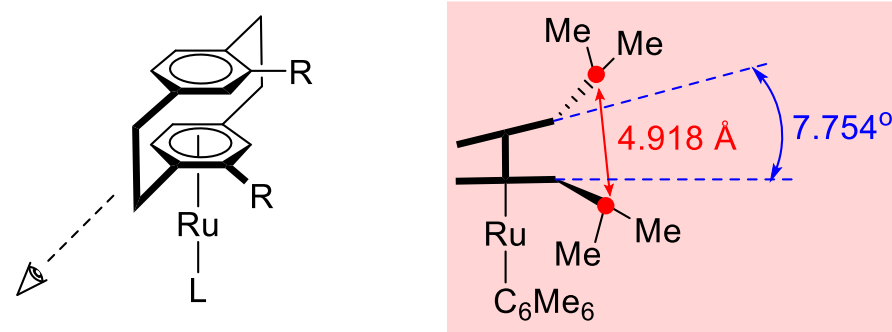

$\left(R_{p}\right)-5 g$

Figure 3. Single crystal structures of chiral arene ruthenium complexes, steric maps, and characteristic distances and angles. 